\title{
Kampo medicine approach for western medicine resistant edema and ascites of cancer patients
}

\author{
Daisuke Tanada, Hiroki Miyawaki, Norihiko Sukenaga, Susumu Nakano, \\ Munetaka Hirose \\ Department of Anesthesiology and pain medicine, Hyogo college of Medicine, Hyogo, Japan
}

[Introduction]

For control of edema and ascites of cancer patients, Western medicine such as diuretics is often used, but in many cases it is difficult to control.

[Objectives]

In our Palliative Care Team, we use a Kampo medicine with water handling effect against western medicine resistant edema and ascites. This time we verified these effects.

\section{[Methods]}

Between December 2015 and November 2017, cases with our team intervening cases using Kampo medicine with water - handling effect against western medicine resistant edema and ascites were examined retrospectively.

[Results]

During that period, 77 cases were used Kampo medicine: $15.4 \%$ of the total intervention (501 cases). Breakdown and effectiveness rates was 36 cases of Goreisan:44.4\% (16/36) , 26 cases of Saireito : $50 \%(13 / 26), 7$ cases of Eppikajutsuto : 14.3\% (1/7), 6 cases of Saireito + Eppikajutsuto : 100\% (6/6) , 2 cases of Goreisan + Eppikajutsuto : 50\% (1/2) .

\section{[Conclusions]}

In this time Kanpo medicine cases were insufficient effect in Western medicine, but good symptom relief was obtained by Kanpo medicine use (an effective rate of $48 \%$ as a whole). In particular, the combined use of Saireito and Eppikajutsuto was effective (effective rate was $100 \%$ ).

We sometimes administered Saireito and Eppikajutsuto from the beginning if it is edema - ascites accompanied by obvious feeling of warmth, but cancer patients often take many medicines, We usually start with Goreisan if it is mild symptoms, we also start with Saireito if it is accompanied by inflammation, and if it is inadequate we will add Eppikajutsuto.

Saireito is a herbal medicine composition of Shosaikoto + Goreisan. Shosaikoto is a drug having anti-inflammatory action against inflammation of the lung / bronchus, liver / upper gastrointestinal tract, brain / meningeal membrane. Goreisan have water-absorbing action through inhibition of AQP4 (Aquaporin 3 - 5) water channel function and Goreisan which is shown to have a selective anti inflammatory effect at AQP expression site. Because of it, Saireito can be expected to be effective for inflammatory edema of these sites.

Eppikajutsuto consists of cooling raw drugs (Ephedra Herb and Gypsum) and water handling raw drugs (Ephedra Herb and Atractylodes Rhizome). Because of these actions, Eppikajutsuto usually use for edema accompanied by exudative inflammation. From the viewpoint of pharmacology, it is said that it shows a strong diuretic action in accordance with the water-harvesting action of tissues will be exerted when as a combination of raw drugs (Poria Sclerotium and Oriental Waterplantain Rhizome) are added to as a combination of raw drugs (Ephedra Herb and Gypsum). Saireito and Eppikajutsuto include those combinations.

There is inflammation in cancer peritonitis, and the tension of the diaphragm coexists along with abdominal distension due to ascites. It is thought that Saireito is more effective than Goreisan because Bupleurum Root has an anti-inflammatory effect and the effect of loosening the tension of the diaphragm.

We would like to continue to use Kampo medicine in conjunction with Western medicine inadequately controlled cases.

\begin{tabular}{|l|l|}
\hline Kampo medicine & Effectiveness \\
\hline Goreisan & $44.4 \%(16 / 36)$ \\
\hline Saireito & $50 \%(13 / 26)$ \\
\hline Eppikajutsuto & $14.3 \%(1 / 7)$ \\
\hline Saireitou+Eppikajutsuto & $100 \%(6 / 6)$ \\
\hline Goreisan+Eppikajutsuto & $50 \%(1 / 2)$ \\
\hline As a whole & $48 \%(37 / 77)$ \\
\hline
\end{tabular}

goreisan (Wu Ling San)

Crude drug composition

Oriental Waterplantain Rhizome, Poria Sclerotium , Bark

Target condition

edema, nausea, vertigo, headache, uremia, diabetes mellitus, overnight drunkenness, jaundice, nephritis, acute gastroenteritis, nausea, acute cystitis, thirst, pollakisuria, nephrotic Syndrome, overnight drunkenness, enteric catarrh, catarrhal gastroenteritis, diarrhoea, dyspeptic diarrhea, vomiting, heat stroke, oliguria, heaviness of head, abdominalgia, Pollakidipsia, infantile diarrhea

Indication goreisan is used for the patients in the stage of Shao yang (lesser yang) and the condition of between Hypo- and Hyperfunction, who has the symptoms such as thirst, reduction of urine output, and sweating

\section{saireito (Chai Ling Tang)}

Crude drug composition

Target condition

Indication

\section{Crude drug}

composition

Target condition

Indication
Bupleurrum Root, Oriental Waterplantain, Rhizome, Pinellia Tuber, Scutellaria Root, Atractylodes Rhizome, Jujube, Polyporus Sclerotium, Ginsen Root, Poria Sclerotium, Glycyrrhiza, Cassia Bark, Ginger

appetite loss, acute gastroenteritis, vomiting, thirst, oliguria, diarrhoea, edema

saireito is used for the patients in the stage of Shao yang (lesser yang) and the condition of between Hypo- and Hyperfunction, who has the symptoms such as stuck feeling in hypochondrium (kyokyo-kuman), thirst, reduced urine volume, and edema

\section{Jia Zhu Tang)}

Ephedra Herb , Gypsum , Ginger , Jujube Glycyrrhiza, Atractylodes Rhizome

edema, nephritis, beriberi, bed-wetting, eczema, knee osteoarthritis, acute conjunctivitis, phlyctenular conjuctivitis, pterygium, nephrotic Syndrome, rheumatoid arthritis, oliguria, pollakisuria

eppikajutsuto is used for the patients in the stage of Shao yang (lesser and the condition of Hyperfunction (excess), who has the symptoms such as thirst, reduction of urine output, and edema 